\title{
Puerto Rico: ¿tres opciones?
}

Maria Patricia Pensado Leglise

$\mathrm{P}$ ara tratar el tema de las últimas elecciones-efectuadas en 1988-y tener un panorama de la situación política en Puerto Rico es necesario hacer antesalgunas precisiones de tipo jurídicoformal e histórico, y de esa manera ubicarnos en el contexto histórico político y en el actual debate político.

Es necesario agregar también que el caso de Puerto Rico difiere de los antes expuestos, debido a la dependencia colonial que guarda con Estados Unidos, razón por la cual en su historia política los referéndums -para decidir la condición jurídica de la isla- han adquirido mayor relevancia y participación del pueblo. Hasta la fecha el estatus de Puerto Rico es un problema sin resolver, que aparece no sólo durante las contiendas electorales, sino en periodos de crisis. Por tanto, la confrontación ideológica en el sistema electoral continúa siendo dominada por la disyuntiva entre "autonomismo" "y "anexionismo". No obstante ser minoritaria, la independencia es una propuesta política que aún sobrevive.

Desde 1952 se establece en Puerto Rico el Estado Libre Asociado (ELA, commonwealth en inglés), bajo el cual "el poder público reside inapelablemente en el pueblo, y así es un Estado libre, pero vinculado a un sistema político más amplio, en asociación federativa o en otra forma que la federal, y por lo tanto no vive independientemente". ${ }^{1}$ Es decir,

1 "Resolución aprobada en la sesión plenaria de la Convención Constituyente de Puerto Rico ce- 
que tiene un margen de decisión en los asuntos internos, sin embargo permanece subordinado al sistema político de Estados Unidos. De esta manera la libertad se tradujo en autogobierno colonial, no en la constitución de un Estado nacional.

Esto se expresa nítidamente en las condiciones de vida y en los derechos políticos y civiles de los puertorriqueños, a pesar de contar con la ciudadanía estadunidense desde 1917, de ser llamados a cumplir el servicio militar, de cantar el himno nacional y saludar la bandera estadunidenses y a aprender inglés obligatoriamente, les es negado el derecho del voto para las elecciones presidenciales y para ser representados de forma proporcional en el Congreso de Estados Unidos; desde 1917 hasta ahora sólo tienen derecho a un representante.

En suma, Puerto Rico carece de soberanía, condición sine qua non que hace del Estado una nación. ${ }^{2}$ Aquélla fue definida desde el siglo XVI por Bodin, como el "poder absoluto y perpetuo de una república, con las connotaciones de 'absoluta', 'perpetua', 'indivisible', 'inalienable' e 'imprescriptible', un poder originario que no depende de otras y que señala también las diferencias entre el derecho privado y el derecho público". En otras palabras, Bodin encuentra la esencia de la soberanía exclusivamente en el "poder de hacer y de abolir las leyes, porque necesariamente absorbe todos los otros poderes y porque como

\footnotetext{
lebrada el día 4 de febrero de 1952", en Manuel Fraga Iribarne (comp.), Las constituciones de Puerto Rico, Ed. Cultura Hispánica, Madrid, 1953, p. 531.

2 "...que en su significado moderno aparece a fines de 1500 junto con el poder de Estado para indicar plenamente el poder estatal único y exclusivo sujeto de la política", Norberto Bobbio y Nicola Matteucci, Diccionario de Politica, Siglo XXI, México, 1982, p. 1535.
}

tal, con sus mandos es la fuerza cohesiva que mantiene unida a toda la sociedad". ${ }^{3}$

Con todo, el estatus jurídico que adopta Puerto Rico en 1952 es la culminación de las luchas políticas que se desatan en el interior de la sociedad puertorriqueña, a partir de la invasión de Estados Unidos en 1898, acerca de la cual Arroyo Barona en su Crónica de Barros escribiría: "Este es el último año de la desgracia colonial y el primero de lo mismo." "

En relación con estas luchas, me referiré brevemente a algunas con el objeto de puntualizar que ni siquiera el ElA fue una concesión gratuita del gobierno de Estados Unidos. En 1929 se plantea el primer referéndum para decidir por votación el estatus de Puerto Rico aunque no se llevó a la práctica; en el lapso de 1931 a 1936 hubo un total de 207 huelgas, las más importantes fueron las que los trabajadores cañeros protagonizaron en 1934; las actividades politicas de los nacionalistas, antiimperialistas y revolucionarios que proponían la independencia total dirigidas por PedroAlvizu Campos; la creación del Partido Comunista en 1934; la matanza de Ponce en 1937, con la cual se inicia la persecución contra todo individuo o grupo que seoponga al dominio estadunidense en la isla; el encarcelamiento de todus los dirigentes del Partido Nacional ${ }^{5}$ que se convierten en proscritos sociales; por último, mencionaré la creación del Partido Popular Democrático en 1938 -escisión del Partido Liberal-dirigido por Luis Muñoz Marín, que aglutina a los sectores

${ }^{3}$ Ibid., p. 1536.

${ }^{4}$ Pedro Arroyo Vivas, Crónica de Barros (Orocovis), años 1825-1960, San Juan de Puerto Rico, 1945.

${ }^{5}$ El Partido Nacional se funda en 1922 y desde un principio se declara independentista; lo dirige en ese entonces Jose Coll y Cuchi. 


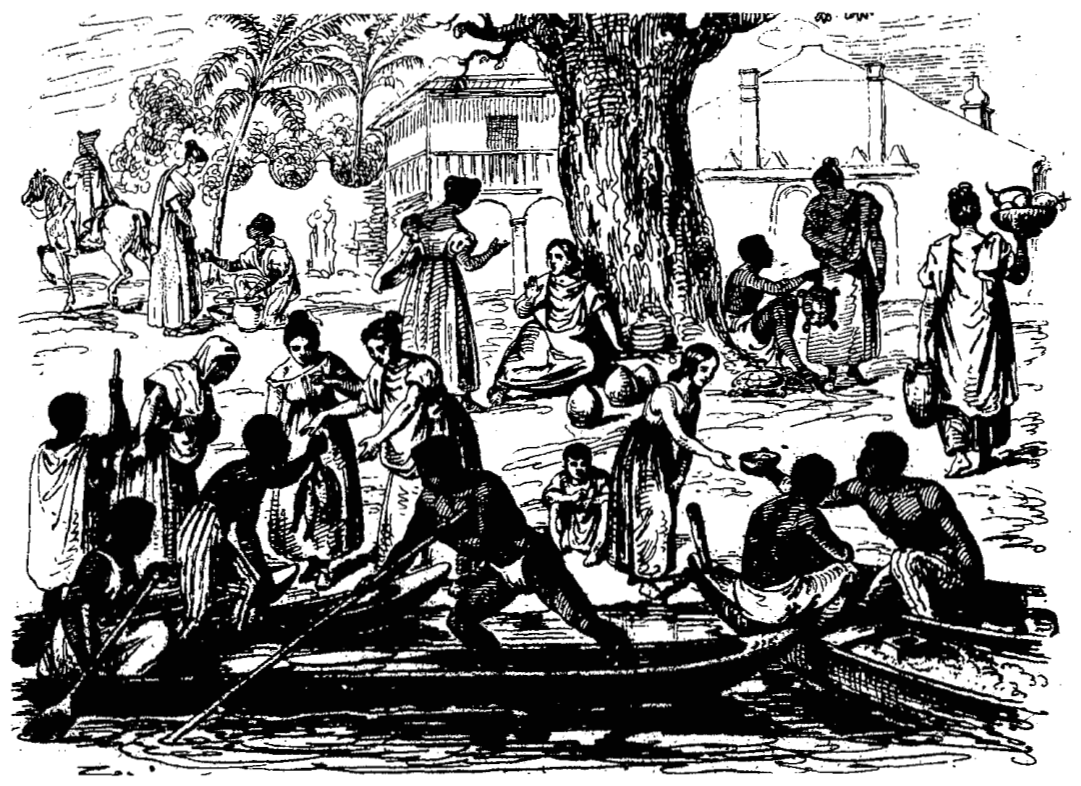

medios urbanos, intelectuales, profesores, herederos de ex hacendados entre otros.

En 1947, el Congreso de Estados Unidos convierte el cargo de gobernador en puesto electivo. Luis Muñoz Marín es el primer gobernador puertorriqueño propuesto en 1948 por Harry Truman, entonces presidente de Estados Unidos. Las elecciones se efectúan cada cuatro años en la misma fecha en que se realizan las presidenciales estadunidenses; este acto de aparente concesión de Estados Unidos no significa más que una mínima retribución a la participación de Puerto Rico en el conflicto bélico mundial y a las excelentes relaciones con el Partido Popular Democrático (PPD), el cual es capaz de crear una serie de expectativas de cambio en las condiciones de vida de los puertorriqueños, sumamente afectadas por la crisis de la década anterior.

En el primer referéndum planteado 21 años antes -en 1951-, 387016 votos aprueban la institución del Estado Libre Asociado, 119169 se oponen y 366900 se abstienen. Al siguiente año se aprueba la nueva Constitución de Puerto Rico; en el artículo primero se aclara que el "poder político emana del pueblo y se ejercerá con arreglo a su voluntad, dentro de los términos del convenio acordado entre el pueblo de Puerto Rico y los Estados Unidos de América". Además: "Tendrá forma republicana y sus poderes legislativo, ejecutivo y judicial según se establecen por esta Constitución, estarán igualmente subordinados a la soberanía del pueblo de Puerto Rico." ${ }^{n}$

6 "Constitución del Estado Libre Asociado de Puerto Rico", en Manuel Fraga Iribarne, op. cit., p. 347. 
La Organización de Naciones Unidas declara que el caso de Puerto Rico encontró solución con la definición del ELA, por tanto exime a Estados Unidos de rendir informes anuales ante los alegatos de que el problema colonial había sido resuelto.

Una vez que el PPD acepta sin condición alguna la alianza con Estados Unidos, desaparece de su espectro político la consigna de " $\mathrm{PPan}$, tierra y libertad!", que anteriormente le había valido la animadversión de los dueños de los enclaves y la simpatía de campesinos y trabajadores. "Los poderes concedidos por el Congreso norteamericano eran suficientes para el desarrollo de su proyecto de clase dentro de los límites de su asociación con el capital norteamericano."

Sin embargo el PPD se mantiene en el poder durante 30 años ininterrumpidos. A partir de las elecciones de 1968 el apoyo del pueblo disminuyó y el PPD. perdió su tradicional primacía electoral.

Es significativo también que para la historia política puertorriqueña el 68 también sea un parteaguas, indudablemente esto tiene que ver con el contexto político internacional: "En 1968, el planeta se inflamó. Parecía que surgía una consigna universal...", escribe CohnBendit. "Queremos al mundo y lo queremos ahora", cantaba Jim Morrison. ${ }^{8}$ La guerra de Vietnam y la lucha por los derechos civiles despertó la conciencia de que el american way of life sólo era para unos pocos elegidos y que desde luego las "minorias étnicas" no pertenecían a ese grupo.

7 Emilio González Díaz, "La lucha de las clases y la política en el Puerto Rico de la década 40: el asenso del PPD", Revista de Ciencias Sociales, Universidad de Puerto Rico, vol. 22, núms. 1-2,1980, p. 66.

${ }^{8}$ Dany Cohn-Bendit, La revolución y nosotros que la quisimos tanto, Anagrama, Barcelona, 1987, p. 12.
Algunos puertorriqueños queestudian en las universidades estadunidenses se suman a las rebeliones estudiantiles, encontrando en ese proceso sus propias reivindicaciones y radicalizando su conciencia nacionalista e independentista. $\mathrm{Si}$ bien esto se vive de una manera diferente en la isla, se percibe también ese estado de ánimo contestatario que cuestiona el orden económico-político, pero que se expresa sobre todo en los movimientos sociales de carácter antisistémico que no son controlados por los partidos políticos; entre ellos podemos mencionar los siguientes: la radicalización del movimiento estudiantil, la resistencia al servicio militar obligatorio y a otras manifestaciones del militarismo, la radicalización de sectores del protestantismo y el catolicismo, las ocupaciones de tierras, la emergencia de un movimiento ambientalista, el desarrollo de un nuevo sindicalismo conformado por sindicatos "independientes" y con un liderazgo de izquierda, la movilización de grupos puertorriqueños emigrantes de Estados Unidos, y el desarrollo de una fuerte corriente crítica en la música, las artes plásticas, la literatura, el teatro y el análisis social. ${ }^{9}$

Por otra parte, es importante mencionar cuáles han sido las condiciones de vida de los puertorriqueños y cuál ha sido el resultado de la aplicación de los diferentes proyectos de industrialización, o mejor dicho de las políticas desarrollistas impuestas por Estados Unidos.

${ }^{9}$ Jorge Rodríguez Beruff, "Puerto Rico en el plano internacional: intereses metropolitanos y reconsolidación del colonialismo", ponencia presentada en el Simposio de Relaciones Internacionales en el Caribe y Centroamérica organizado por Clacsoy la Universidad de Puerto Rico, San Juan de Puerto Rico, 26-28 de enero, 1988, El Caribe Contemporáneo, núm. 17, jul.-dic., 1988, México, p. 38. 
Durante la segunda posguerra los países de la cuenca del Caribe son objeto de dos tendencias principales en el desarrollo económico. Por un lado, la modernización y diversificación de la producción primaria (agricultura y minería). Por otra parte, del impulso a un proceso de industrialización sustitutiva de importaciones, centrado en industrias de mano de obra intensiva. En principio estas industrias debieron orientarse hacia la producción de manufacturas para los mercados locales y regionales, pero durante la década de los setenta una parte cada vez mayor de esta producciôn, controlada por intereses extranjeros, se orientó hacia la exportación. De esta forma proliferan en la última década las industrias de procesamiento de importación-exportación o maquiladoras. ${ }^{10}$

La aplicación de las políticas desarrollistas durante la década de los cuarenta logró el crecimiento económico a costa de la expansión del sector terciario, particularmente empleos públicos y las actividades de la guerra. Las otras actividades eran producto de la instalación de industrias básicas orientadas al mercado local tanto en sus insumos como en su producción (por ejemplo, empresas que producían botellas para la industria del ron, cajas de cartón, fábricas de cemento, de cerámicas, de materiales de construcción, de zapatos y pieles, y una planta textil) En esta época también se decreta la primera reforma agraria que, "en términos formales se proponía la diversificación de la agricultura, la ruptura del enclave monoproductor y el dominio explotador delos trabajadores de la tierra y la devolución a éstos de lo que en justicia era suyo: la tierra que traba-

${ }^{10}$ Emilio Pantojas, "La iniciativa de la cuenca del Caribe y Puerto Rico", El Caribe Contemporáneo, núm.12, dic., 1986, México, p. 55. jaban". ${ }^{11}$ Se otorgarían 500 acres a toda corporación ("persona jurídica") y disponía el procedimiento para la expropiación o compra de las tierras en exceso de esa cantidad. Sin embargo, la oposición de las corporaciones azucareras y la ausencia de poder político autónomo en la rama ejecutiva impedirán el desarrollo efectivo de la misma. Esto contribuye a que no se pueda evitar la crisis agrícola permanente.

Más tarde, a principios de la década de los ochenta, el presidente Ronald Reagan anuncia en febrero de 1982 la Iniciativa de la Cuenca del Caribe (ICC), misma que se convirtió en ley al año siguiente. En lo que a Puerto Rico se refiere, tal iniciativa no iba a cambiar el tipo de industrialización que anteriormente había prevalecido, es decir, si "no promueve el eslabonamiento entre las distintas fases del proceso productivo es incapaz de estimular el desarrollo de una industria local fuerte, con capacidad para generar ahorro interno [excedentes de capital] suficiente para la expansión económica sostenida y reducir así la dependencia de fuentes externas para el financiamiento de inversiones". Este tipo de desarrollo industrial tuvo como efecto que la economía puertorriqueña en el sector manufacturero, dejara en manos del capital norteamericano las decisiones fundamentales sobre inversión y productos en el sector más dinámico de la economía del país. ${ }^{12}$

De esta manera, la ICC estimula el mismo tipo de desarrollo del sector industrial que venía creciendo en décadas pasadas: la maquila.

${ }^{11}$ Emilio Pantojas García, "Desarrollismo y lucha de clases: los límites del proyecto populista en Puerto Rico durante la década del cuarenta", Revista de Ciencias Sociales, Universidad de Puerto Rico, vol. 24, núm. 4, 1985, p. 371.

${ }^{12}$ Emilio Pantojas Garcia, op. cit., p. 59. 
Actualmente las actividades "productivas" que se han desarrollado en Puerto Rico, siguen siendo casi las mismas: en primer lugar las actividades del turismo, manufactura en la rama farmacéutica y en la petroquímica. La tasa de desempleo es aproximadamente de $20 \%$, sin embargo $50 \%$ de las familias de la isla dependen para su subsistencia del programa de subsidios para la alimentación; 2000000 de puertorriqueños viven en Estados Unidos (la mayoría en estado de pobreza). Además la deuda per cápita que han contraído los puertorriqueños con los acreedores estadunidenses: es de 18 billones de dólares por 3200000 habitantes.

Al igual que en otros países, en Puerto Rico el costo de "la urbanización y la industrialización extensivas conllevan consecuencias laterales indeseadas: agotamiento de recursos no renovables, deforestación y cambios climáticos, contami- nación ambiental, liquidación de especies animales enteras, degradación delas zonas rurales próximas a las ciudades, aparición de entornos urbanos inhabitables", ${ }^{13}$ y sus colaterales consecuencias sociales; entre estas últimas se encuentra el total abandono del campo y el desapego a él por parte de las nuevas generaciones. En una nota del Washington Post del 7 de marzo de 1989 se dice que en la actualidad de $85 \%$ a $90 \%$ de la alimentación es importada de Estados Unidos y de los países vecinos. Puerto Rico es el segundo país, después de Canadá, consumidor de productos básicos per cápita provenientes de Estados Unidos; el cual envía anualmente para asistencia a la isla más de 10 billones de dólares.

${ }^{13}$ Ludolfo Paramio, Tras el diluvio. La izquierda ante el fin de siglo, Siglo XXI, México, 1989, 2a. ed., p. 223.

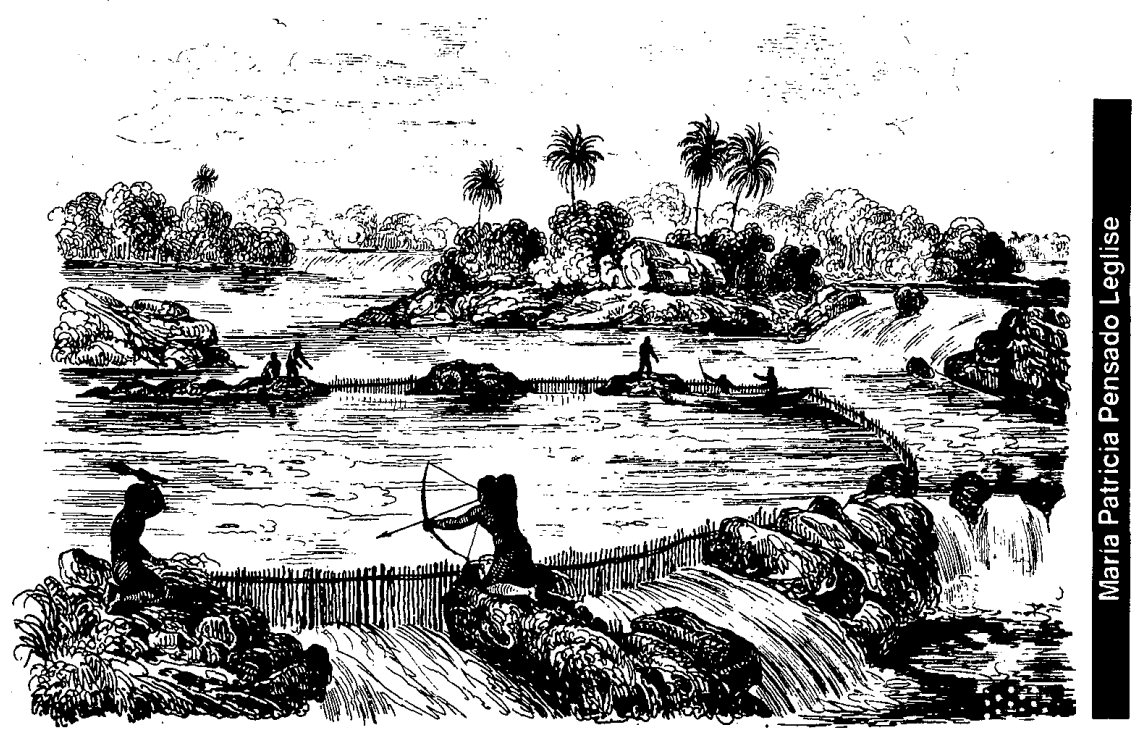


Si bien "la opresión no hace bien a nadie" -según comenta Dulce Ma. Pascual-, en el ámbito político hace aún más complicada la participación social de los individuos, para quienes los pequeños privilegios económicos (bonos para comida, asistencia social entre otros) se convierten en diques que impiden el flujo natural de la actividad política colectiva y el desarrollo de las organizaciones políticas y sociales.

Desde 1968 el PPD y el partido Nuevo Progresista (PNP) se han alternado en el poder con un margen mínimo de diferencia en el total de la votación. En las últimas elecciones -6 de noviembre de 1988- Rafael Hernández Colón, candidato del PPD es reelecto por tercera vez; ganó con 865169 votos (48.7\%), le siguió Baltasar Corrada del Río, candidato del PNP con 83230 votos $(45.8 \%)$ y por último, Rubén Berrios Martínez, candidato del Partido Independentista Puertorriqueño (PIP) con 96222 votos (5.4\%). Estas cifras son del total de votos válidos emitidos.

En cuanto al estudio sobre el comportamiento electoral de las distintas clasesy grupos sociales, todavía hace falta profundizar; la información que existe es escasa y ambigua para poder hacer alguna reflexión teórica. Los estudios anteriores revelan la simpatía de sectores rurales y populares por el PPD. Sin embargo, ante una irremediable descomposición de la "sociedad rural", es dificil imaginar que continúe siendo una de sus bases de apoyo. También resulta simplista considerar que el gran número de subempleadosy desempleados que sobreviven por la "ayuda económica" y los servicios prestados por Estados Unidos son leales al PNP y a defender la condición de estado, como ya ha sido mencionado por

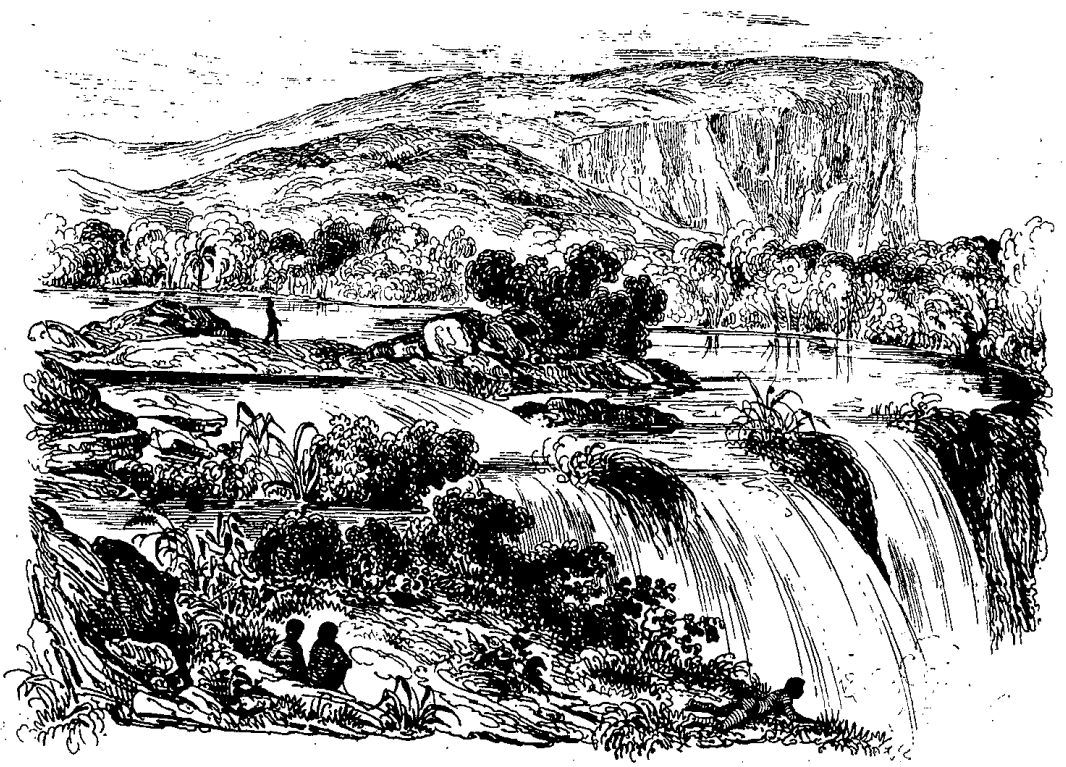


los dirigentes de los partidos. Por otra parte, tampoco son únicamente los intelectuales y los estudiantes de izquierda quienes se pronuncian por la independencia. Y, finalmente la clase política puertorriqueña no está escindida en dos posicionesirreconciliables, como algunos se empeñan en destacar, al referirse a una pugna entre elites viejas frente a las modernas, cada vez mas proestadunidenses. Tal vez haga falta matizar y definir acuciosamente, tanto las posiciones $\mathrm{u}$ opciones que plantean para la vida política y económica futura de Puerto Rico, como conocer la lealtad de sus sustentantes.

A su vez los tres candidatos de los tres partidos mayoritarios representan las tres posiciones que ofrecen diferentes opciones para definir el status de la isla, para lo cual se propone un referéndum.

El último referéndum se llevó a cabo en 1967, en esa ocasión la votación de la mayoría fue por mantener el ELA (425132 votos); por la categoría de estado federal, es decir convertir a Puerto Rico en el estado quincuagésimo primero de Estados Unidos (274 312 votos); por la independencia ( 4 248). Cabe señalar que parte de la izquierda y de la disidencia puertorriqueña optó por llamar al abstencionismo y de esa manera boicotear el referéndum como protesta; esto explica el número de abstenciones en ese proceso (360 065).

Desde su discurso inaugural del 4 de enero de 1989, el gobernador electo Hernández Colón prometió resolver la condición de la isla de una vez por todas y mencionó las ventajas de la unión permanente con Estados Unidos, pero como Estado Libre Asociado, aplicando algunas reformas que otorguen mayor poder de decisión y más autonomía al gobierno interno de la isla, manteniendo la nacionalidad estadunidense, las rela- ciones comerciales, el dólar en circulación y los acuerdos de "defensa". Exigirán continuar con el proceso de industrialización con mayor eficiencia para evitar la inmigración de mano de obra barata de sus vecinos caribeños y tomar decisiones en los asuntos internacionales.

El 17 de enero los dirigentes de los tres partidos mayoritarios se reúnen para tratar el tema de redefinir las relaciones político-económicas con Estados Unidos. Baltasar Corrada del Río del PNP se pronuncia por la categoría de estado, argumentando que esto significaría tomar decisiones en la vida interna de la metrópoli; por ejemplo, se obtendría el derecho a votar en las elecciones presidenciales, elegir dos senadores y tres o cuatro representantes en el Congreso, el derecho exclusivo para administrar sus recursos naturales, mantener el español como idioma principal con la finalidad de lesionar menos su cultura aceptando su herencia española. La tercera posición es la de Rubén Berrios Martínez, dirigente y candidato del Partido Independentista Puertorriqueño, que propone el proyecto independentista, la opción minoritaria.

George Bush, presidente electo de Estados.Unidos, al presentar su primer presupuesto al Congreso de 1989 hizo alusión personal al a poyo que brindará a los puertorriqueños que propongan la condición de estado, sustentando el derecho que tienen los 2000000 de votantes a decidir el cambio de estatus. Como resulta obvio, la declaración del presidente estadunidense suscitó el debate en el Congreso y los partidos.

En una entrevista, Rubén Berrios declaró que es la primera vez que Estados Unidos quiere sentarse a negociar con ellos. Uno de los problemas más graves, señala Berrios, es que "la isla 


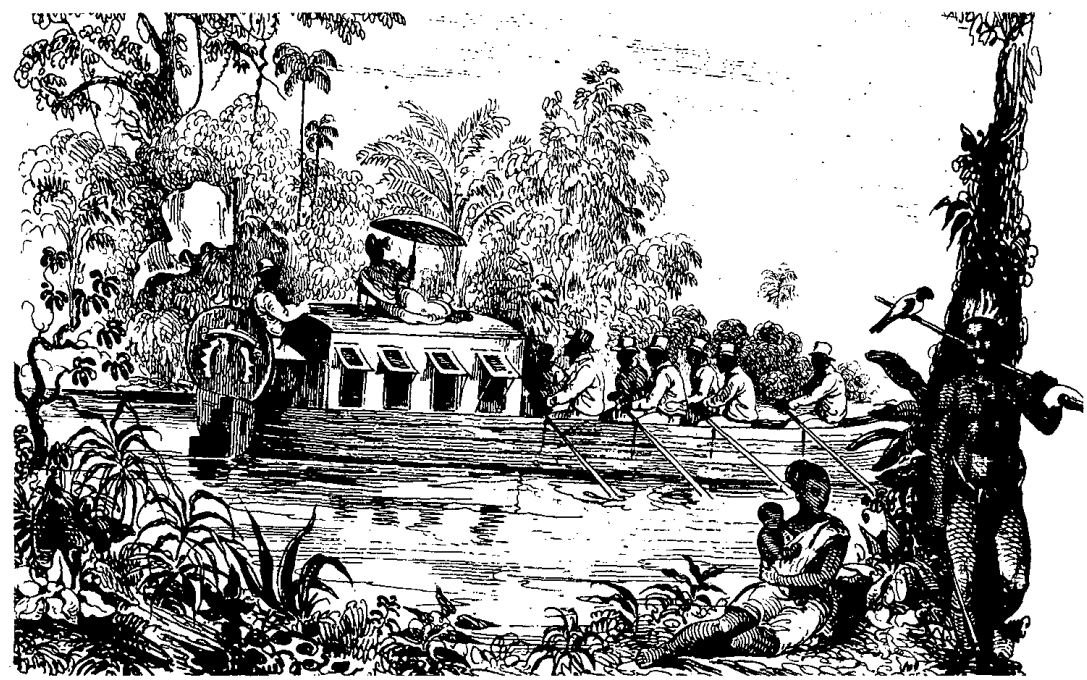

completa ahora tiene mentalidad de ghetto"; opina también que Estados Unidos sólo estará dispuesto a hacer algunos cambios o reformas manteniendo el actual estatus; esto los favorece más que en el nivel económico (libre de impuesto), en el político y estratégico, pues mantienen la isla como una base militar. Agregó también que es importante que la ONU ruelva a considerar el problema colonial que vive Puerto Rico y cambie la definición de territorio autónomo por colonia. ${ }^{14}$

El senador J. Bennett Johnston -demócrata de Louisiana- presidente del Comité de Energía y Recursos Naturales del Senado, convocó a una reunión entre representantes puertorriqueños y miembros del Senado para discutir y proponer se efectúe el referéndum en el verano de 1991.

${ }^{14}$ Los Angeles Times, 21 de abril de 1989, en ISLA, vol. xxxvin, núm. 4.
Durante el desarrollo de este encuentro, se suscitaron en Puerto Rico expresiones de rechazo al referéndum, ocurrieron dos explosiones de bombas en el Chase Manhattan Bank, en la población de Caguas y en el Bank of Boston, en Bayamón. ${ }^{15}$ Mientras se manifestaban miles de personas que salieron a la calle cantando y ondeando banderas puertorriqueñas, quemando la estadunidense y gritando: "iSí a la república, no a la colonia!", los senadores J. Bennett Johnston, James A. McClure-republicano de Idahoy Daniel P. Moynihan -demócrata de Nueva York-escuchaban testimonios de los adeptos de cada una de las tres opciones. Algunos manifestantes dijeron que se habían reunido aproximadamente 80000 personas, mientras que la televisión puertorriqueña apuntó que eran menos de $12000 . .^{16}$

${ }^{15}$ Miami Herald, 17 y 20 de junio de 1989, en ISLA, vol. XXXVIII, núm. 6.

${ }^{16}$ New York Times, 21 de junio de 1989, en ISLA, vol. xxxviII, núm. 6. 


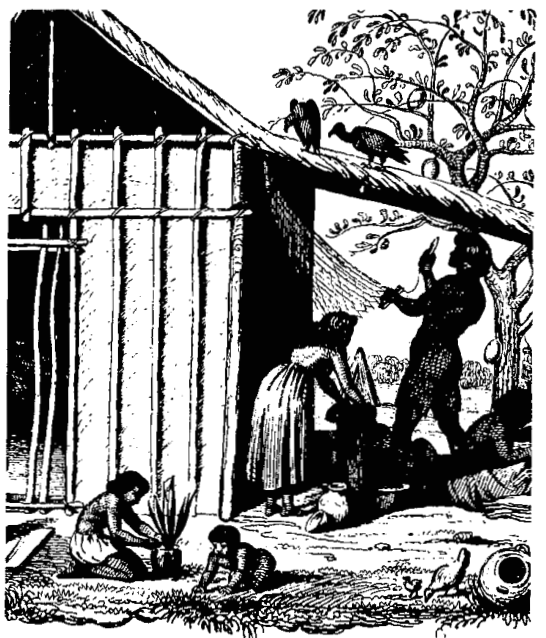

Por las declaraciones del senador James A. McClure, representante del Partido Republicano de Estados Unidos, conceder la categoría de estado no significa correr riesgos, sobre todo cuando se cuenta con la experiencia de Alaska y Hawai que se convierten en estados federados de Estados Unidos en 1959.

A pesar de todos los argumentos en pro y en contra, el estado significa un desafío a Estados Unidos, debido a que pondría freno a la explotación irracional de los recursos naturales que hace la industria, y obligaría a aplicar las mismas medidas de seguridad que en Estados Unidos, es decir a controlar las industrias altamente contaminantes, a pagar salarios equivalentes a los de los trabajadores estadunidenses, a otorgar los mismos derechos en todo lo que respecta a la asistencia social, lo cual implica a su vez una reconversión industrial, y aplicar programas dirigidos a erradicar la pobreza, de lo contrario Puerto Rico se convertirá en el estado más pobre deEstados Unidos.
Por otra parte también significará vencer el miedo de aceptar la "otredad cultural"; Guillermo Gómez Peña lo define como el temor a que

ocupemos "su país", sus trabajos, sus suburbios, sus universidades, su mundo de arte. Ellos piensan que "nosotros" somos todo un paquetazo que incluye un idioma español impreciso, unas artes rarísimas, comportamientos tumultuarios, drogas, indocumentados y terrorismo en potencia. No se dan cuenta que sus temores les han sido implantados como una forma de control político: que el temor es la fuente misma dela violencia endémica que afecta esta sociedad. ${ }^{17}$

En lo que respecta a la alternativa de mantener la condición de ELA pero con reformas, expresa las aspiraciones de una clase dominante que no acaba de serlo y que desea una mayor cuota de poder político y económico.

Finalmente, la última opción, al parecer la más débil y que además no es representativa de todos los grupos que defienden la independencia de Puerto Rico, insiste en que Estados Unidos no sea solamente el que decida de manera unilateral el quehacer político y el futuro de la isla; están convencidos de que el poder de Estados Unidos se ha implantado en la vida cotidiana de los puertorriqueños, es decir, que el dictado omnipresente de la cultura de consumo, de la producción, de la publicidad, del comercio, refuerzan el carácter ilusorio de las libertades que no se fundan en la responsabilidad concreta del individuo, privándolo del derecho de disentir e imaginar que Puerto Rico puede llegar a ser un país libre y soberano si se lo proponen.

17 Guillermo Gómez Peña, "Paradigmas culturales", La Jornada Semanal, núm. 37, 25 de febrero de 1990, México, p. 34. 